\title{
SS-Injective Modules and Rings
}

\author{
Akeel Ramadan Mehdi \\ Department of Mathematics, \\ College of Education, , \\ University of Al-Qadisiyah, Iraq \\ Email: akeel.mehdi@qu.edu.iq
}

\author{
Adel Salim Tayyah \\ Department of Mathematics \\ College of Computer Science and Information Technology, \\ University of Al-Qadisiyah, Iraq \\ Email: adel.tayh@qu.edu.iq
}

\begin{abstract}
We introduce and investigate SS-injectivity as a generalization of both soc-injectivity and small injectivity. A right module $M$ over a ring $R$ is said to be SS- $N$-injective (where $N$ is a right $R$-module) if every $R$-homomorphism from a semisimple small submodule of $N$ into $M$ extends to $N$. A module $M$ is said to be SS-injective (resp. strongly SSinjective), if $M$ is SS-R-injective (resp. SS- $N$-injective for every right $R$-module $N)$. Some characterizations and properties of (strongly) SS-injective modules and rings are given. Some results on soc-injectivity are extended to SSinjectivity.
\end{abstract}

Key words and phrases: Small Injective rings (modules); Soc-Injective rings (modules); SS-Injective rings (modules); Perfect rings; quasi-Frobenius rings.

Mathematics subject classification: Primary: 16D50, 16D60, 16D80; Secondary: 16P20, 16P40, 16L60.

\section{Introduction}

Throughout this paper, $R$ is an associative ring with identity, and all modules are unitary right $R$-modules. For a right $R$-module $M$, we write $\operatorname{soc}(M), J(M)$, $Z(M), Z_{2}(M), E(M)$ and $\operatorname{End}(M)$ for the socle, the Jacobson radical, the singular submodule, the second singular submodule, the injective hull and the endomorphism ring of $M$, respectively. Also, we use $S_{r}, S_{\ell}, Z_{r}, Z_{\ell}, Z_{2}^{r}$ and $J$ to indicate

the right socle, the left socle, the right singular ideal, the left singular ideal, the right second singular ideal, and the Jacobson radical of $R$, respectively. For a submodule $N$ of $M$, we write $N \subseteq{ }^{e s s} M, N \ll M$, $N \subseteq \oplus$, and $N \subseteq^{\max } M$ to indicate that $N$ is an essential submodule, a small submodule, a direct summand, and a maximal submodule of $M$, respectively. If $X$ is a subset of a right $R$-module $M$. The right (resp. left) annihilator of $X$ in $R$ is denoted by $r_{R}(X)$ (resp. $\left.l_{R}(X)\right)$. If $M=R$, we write $r_{R}(X)=r(X)$ and $l_{R}(X)=l(X)$.

Let $M$ and $N$ be right $R$-modules, $M$ is called soc- $N$ injective if every $R$-homomorphism from the $\operatorname{soc}(N)$ into $M$ extends to $N$. A right $R$-module $M$ is called socinjective, if $M$ is soc- $R$-injective. A right $R$-module $M$ is called strongly soc-injective, if $M$ is soc- $N$-injective for all right $R$-module $N$ [1].

Recall that a right $R$-module $M$ is called mininjective [2] ( resp. small injective [3], principally small injective
[4]) if every $R$-homomorphism from any simple (resp. small, principally small) right ideal to $M$ extends to $R$. A ring $R$ is called right mininjective (resp. small injective, principally small injective) ring, if it is right mininjective (resp. small injective, principally small injective) as right $R$-module. A ring $R$ is called right Kasch if every simple right $R$-module embeds in $R$ (see for example [5]). Recall that a $\operatorname{ring} R$ is called semilocal if $R / J$ is a semisimple [6]. Also, a ring $R$ is said to be right perfect if every right $R$-module has projective cover. Recall that a ring $R$ is said to be quasi-Frobenius (or $Q F$ ) ring if it is right (or left) artinian and right (or left) self-injective; or equivalently, every injective right $R$-module is projective.

In this paper, we introduce and investigate the notions of SS-injective and strongly SS-injective modules and rings. Examples are given to show that the (strong) SS-injectivity is distinct from that of mininjectivity, principally small injectivity, small injectivity, simple J-injectivity, and (strong) socinjectivity. Some characterizations and properties of (strongly) SS-injective modules and rings are given.

In Section 2, we give some basic properties of SSinjective modules. For examples, we prove that a ring $R$ is right universally mininjective if and only if every simple right ideal is SS-injective. We also prove that if $M$ is projective right $R$-module, then every quotient of an SS- $M$-injective right $R$-module is SS- $M$-injective if and only if $\operatorname{soc}(M) \cap J(M)$ is projective. We show that 
if every simple singular right $R$-module is SS-injective, then $S_{r}$ is projective and $r(a) \subseteq \oplus R_{R}$ for all $a \in S_{r} \cap J$.

In Section 3, we show that a right $R$-module $M$ is strongly SS-injective if and only if every small submodule $A$ of a right $R$-module $N$, every $R$ homomorphism $\alpha: A \rightarrow M$ with $\alpha(A)$ semisimple extends to $N$. In particular, $R$ is semiprimitive if every simple right $R$-module is strongly SS-injective, but not conversely. We also prove that if $R$ is a right perfect ring, then a right $R$-module $M$ is strongly soc-injective if and only if $M$ is strongly SS-injective. A results ([1, Theorem 3.6 and Proposition 3.7]) are extended. We prove that a ring $R$ is right artinian if and only if every direct sum of strongly SS-injective right $R$-modules is injective, and $R$ is $Q F$ ring if and only if every strongly $\mathrm{SS}$-injective right $R$-module is projective.

In Section 4, we extend the results ([1, Proposition 4.6 and Theorem 4.12]) from a soc-injective ring to an SS-injective ring ( see Proposition 4.9 and Corollary 4.10).

In Section 5, we show that a ring $R$ is $Q F$ if and only if it is strongly SS-injective and right noetherian with essential right socle if and only if it is strongly SSinjective, $l\left(J^{2}\right)$ is countable generated left ideal, $S_{r} \subseteq{ }^{e s s} R_{R}$, and the chain $r\left(x_{1}\right) \subseteq r\left(x_{2} x_{1}\right) \subseteq \cdots \subseteq$ $r\left(x_{n} x_{n-1} \ldots x_{1}\right) \subseteq \cdots$ terminates for every infinite sequence $x_{1}, x_{2}, \ldots$ in $R$ (see Theorem 5.9 and Theorem 5.11). Finally, we prove that a ring $R$ is $Q F$ if and only if $R$ is strongly left and right SS-injective, left Kasch, and $J$ is left $t$-nilpotent (see Theorem 5.14), extending a result of I. Amin, M. Yousif and N. Zeyada [1, Propostion 5.8] on strongly soc-injective rings.

General background material can be found in [7], [8] and [9].

\section{SS-Injective Modules}

Definition 2.1. Let $N$ be a right $R$-module. A right $R$-module $M$ is said to be SS- $N$-injective, if for any semisimple small submodule $K$ of $N$, any right $R$ homomorphism $f: K \rightarrow M$ extends to $N$. A module $M$ is said to be SS-quasi-injective if $M$ is SS- $M$-injective. $M$ is said to be SS-injective if $M$ is SS-R-injective. A ring $R$ is said to be right $\mathrm{SS}$-injective if the right $R$ module $R_{R}$ is SS-injective.

Definition 2.2. A right $R$-module $M$ is said to be strongly SS-injective if $M$ is SS- $N$-injective, for all right $R$-module $N$. A ring $R$ is said to be strongly right SS-injective if the right $R$-module $R_{R}$ is strongly SSinjective.

\section{Example 2.3.}

(1) Every soc-injective module is SS-injective, but not conversely (see Example 5.7).

(2) Every small injective module is SS-injective, but not conversely (see Example 5.5).
(3) Every $\mathbb{Z}$-module is SS-injective. In fact, if $M$ is a $\mathbb{Z}$-module, then $M$ is small injective (by [3, Theorem 2.8) and hence it is SS-injective.

(4) The two classes of principally small injective rings and SS-injective rings are different ( see [5, Example 5.2], Example 4.4 and Example 5.5).

(5) Every strongly soc-injective module is strongly SS-injective, but not conversely (see Example 5.7).

(6) Every strongly SS-injective module is SSinjective, but not conversely (see Example 5.6).

Theorem 2.4. The following statements hold:

(1) Let $N$ be a right $R$-module and let $\left\{M_{i}: i \in I\right\}$ be a family of right $R$-modules. Then the direct product $\prod_{i \in I} M_{i}$ is SS-N-injective if and only if each $M_{i}$ is SS-N-injective, $i \in I$.

(2) Let $M, N$ and $K$ be right $R$-modules with $K \subseteq N$. If $M$ is SS- $N$-injective, then $M$ is SS- $K$-injective.

(3) Let $M, N$ and $K$ be right $R$-modules with $M \cong N$. If $M$ is SS- $K$-injective, then $N$ is SS- $K$-injective.

(4) Let $M, N$ and $K$ be right $R$-modules with $K \cong N$ and $M$ is SS- $K$-injective. Then $M$ is SS- $N$ injective.

(5) Let $M, N$ and $K$ be right $R$-modules with $N$ is a direct summand of $M$. If $M$ is SS- $K$-injective, then $N$ is SS- $K$-injective.

Proof. Clear.

\section{Corollary 2.5 .}

(1) If $N$ is a right $R$-module, then a finite direct sum of $\mathrm{SS}-N$-injective modules is again $\mathrm{SS}-N$ injective. Moreover, a finite direct sum of SSinjective (resp. strongly SS-injective) modules is again SS-injective (resp. strongly SS-injective).

(2) A direct summand of an SS-quasi-injective (resp., SS-injective, strongly SS-injective) module is again SS-quasi-injective (resp., SS-injective, strongly SS-injective).

Proof. (1) Take the index $I$ to be a finite set and apply Theorem 2.4 (1).

(2) This follows from Theorem 2.4 (5).

Proposition 2.6. Every SS-injective right $R$-module is a right mininjective. 
Proof. Let $I$ be a simple right ideal of $R$. By [10, Lemma 3.8, p. 29] we have that either $I$ is nilpotent or a direct summand of $R$. If $I$ is a nilpotent, then $I \subseteq J$ by [11, Corollary 6.2.8, p. 181] and hence $I$ is a simple small right ideal of $R$. Thus every SS-injective right $R$ module is right mininjective.

It easy to prove the following proposition.

Proposition 2.7. Let $N$ be a right $R$-module. If $J(N)$ is a small submodule of $N$, then a right $R$-module $M$ is SS- $N$-injective if and only if any $R$-homomorphism $f: \operatorname{soc}(N) \cap J(N) \rightarrow M$ extends to $N$.

Proposition 2.8. Let $N$ be a right $R$-module and $\left\{A_{i}: i=1,2, \ldots, n\right\}$ be a family of finitely generated right $R$-modules. Then $N$ is SS- $\bigoplus_{i=1}^{n} A_{i}$-injective if and only if $N$ is SS- $A_{i}$-injective, for all $i=1,2, \ldots, n$.

Proof. $(\Rightarrow)$ This follows from Theorem 2.4 ((2), (4)).

$(\Leftarrow)$ By [12, Proposition (I.4.1) and Proposition (I.1.2), p. 28 and 16] we have $\operatorname{soc}\left(\bigoplus_{i=1}^{n} A_{i}\right) \cap J\left(\bigoplus_{i=1}^{n} A_{i}\right)=$ $(\operatorname{soc} \cap J)\left(\bigoplus_{i=1}^{n} A_{i}\right)=\bigoplus_{i=1}^{n}(\operatorname{soc} \cap J)\left(A_{i}\right)=$ $\bigoplus_{i=1}^{n}\left(\operatorname{soc}\left(A_{i}\right) \cap J\left(A_{i}\right)\right)$. For $j=1,2, \ldots, n$ consider the following diagram:

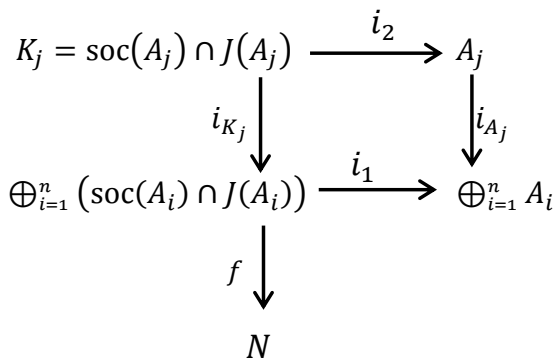

where $i_{1}, i_{2}$ are inclusion maps and $i_{K_{j}}, i_{A_{j}}$ are injection maps. By hypothesis, there exists an $R$-homomorphism $h_{j}: A_{j} \rightarrow N$ such that $h_{j} i_{2}=f i_{K_{j}}$, also there exists exactly one $R$-homomorphism $h: \bigoplus_{i=1}^{n} A_{i} \rightarrow N$ satisfying $h_{j}=h i_{A_{j}}$ by [8, Theorem 4.1.6 (2), p. 83]. Thus $f i_{K_{j}}=h_{j} i_{2}=h i_{A_{j}} i_{2}=h i_{1} i_{K_{j}}$ for all $j=$ $1,2, \ldots, n$. Let $\left(a_{1}, a_{2}, \ldots, a_{n}\right) \in \bigoplus_{i=1}^{n}\left(\operatorname{soc}\left(A_{i}\right) \cap\right.$ $\left.J\left(A_{i}\right)\right)$, thus $a_{j} \in \operatorname{soc}\left(A_{j}\right) \cap J\left(A_{j}\right)$, for all $j=1,2, \ldots, n$, and

$f\left(\left(a_{1}, a_{2}, \ldots, a_{n}\right)\right)=f\left(i_{K_{1}}\left(a_{1}\right)\right)+f\left(i_{K_{2}}\left(a_{2}\right)\right)+\cdots+$ $f\left(i_{K_{n}}\left(a_{n}\right)\right)=\left(h i_{1}\right)\left(\left(a_{1}, a_{2}, \ldots, a_{n}\right)\right)$. Thus $f=h i_{1}$ and the proof is complete.

\section{Corollary 2.9.}

(1) Let $1=e_{1}+e_{2}+\cdots+e_{n}$ in $R$, where the $e_{i}$ are orthogonal idempotents. Then $M$ is SS-injective if and only if $M$ is $\mathrm{SS}-e_{i} R$-injective for every $\mathrm{i}=1,2, \ldots, n$.

(2) For idempotents $e$ and $f$ of $R$. If $e R \cong f R$ and $M$ is SS-eR-injective, then $M$ is SS- $f R$-injective.
Proof. (1) From [7, Corollary 7.3, p. 96], we have $R=\bigoplus_{i=1}^{n} e_{i} R$, thus it follows from Proposition 2.8 that $M$ is SS-injective if and only if $M$ is SS- $e_{i} R$-injective for all $1 \leq i \leq n$.

(2) This follows from Theorem 2.4 (4).

Corollary 2.10. A right $R$-module $M$ is SS-injective if and only if $M$ is SS- $P$-injective, for every finitely generated projective right $R$-module $P$.

Proof. By Proposition 2.8 and Theorem 2.4 ((2), (4)).

Proposition 2.11. The following statements are equivalent for a right $R$-module $M$ :

(1) Every right $R$-module is SS- $M$-injective.

(2) Every simple submodule of $M$ is SS- $M$-injective.

(3) $\operatorname{soc}(M) \cap J(M)=0$.

Proof. $(1) \Rightarrow(2)$ and $(3) \Rightarrow(1)$ are obvious. $(2) \Rightarrow(3) \quad$ Assume that $\operatorname{soc}(M) \cap J(M) \neq 0$, thus $\operatorname{soc}(M) \cap J(M)=\bigoplus_{i \in I} x_{i} R$ where $x_{i} R$ is a simple small submodule of $M$, for each $i \in I$. Therefore $x_{i} R$ is SS- $M$ injective for each $i \in I$ by hypothesis. For any $i \in I$, the inclusion map from $x_{i} R$ to $M$ is split, so we have that $x_{i} R \subseteq \subseteq^{\oplus} M$. Since $x_{i} R$ is small submodule of $M$, it follows that $x_{i} R=0$ and hence $x_{i}=0$ for all $i \in I$ and this a contradiction.

A ring $R$ is called right universally mininjective ring if it is satisfies the condition $S_{r} \cap J=0$ (see for example [2, Lemma 5.1]).

Corollary 2.12. The following statements are equivalent for a ring $R$ :

(1) $R$ is right universally mininjective.

(2) Every right $R$-module is SS-injective.

(3) Every simple right ideal is SS-injective.

Proof. By Proposition 2.11.

Theorem 2.13. (SS-Baer's condition) The following statement are equivalent for a ring $R$ :

(1) $\quad M$ is an SS-injective right $R$-module.

(2) If $S_{r} \cap J=A \oplus B$, and $\alpha: A \rightarrow M$ is an $R$ homomorphism, then there exists $m \in M$ such that $\alpha(a)=m a$ for all $a \in A$ and $m B=0$.

\section{Proof. Clear.}

Theorem 2.14. If $M$ is a projective right $R$-module, then the following statements are equivalent:

(1) Every quotient of an SS-M-injective right $R$ module is SS- $M$-injective.

(2) Every quotient of a soc- $M$-injective right $R$-module is SS- $M$-injective. 
(4) Every quotient of an injective right $R$-module is SS- $M$-injective.

(5) Every sum of two SS- $M$-injective submodules of a right $R$-module is SS- $M$-injective.

(6) Every sum of two soc- $M$-injective submodules of a right $R$-module is SS- $M$-injective.

(7) Every sum of two injective submodules of a right $R$-module is SS- $M$-injective.

(8) Every semisimple small submodule of $M$ is projective.

(9) Every simple small submodule of $M$ is projective.

(10) $\operatorname{soc}(M) \cap J(M)$ is projective.

Proof. $(1) \Rightarrow(2) \Rightarrow(3),(4) \Rightarrow(5) \Rightarrow(6)$ and $(9) \Rightarrow(7) \Rightarrow(8)$ are obvious.

$(8) \Rightarrow(9)$ Since $\operatorname{soc}(M) \cap J(M)$ is a direct sum of simple submodules of $M$ and since every simple in $J(M)$ is small in $M$, thus $\operatorname{soc}(M) \cap J(M)$ is projective.

(3) $\Rightarrow$ (7) Let $D$ and $N$ be right $R$-modules and consider the diagram:

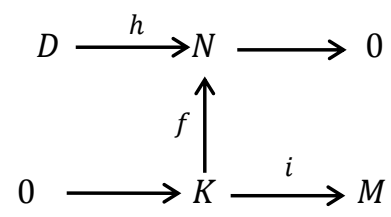

where $K$ is a semisimple small submodule of $M, h$ is a right $R$-epimorphism, $f$ is a right $R$-homomorphism, and $i$ is the inclusion map. We can take $D$ to be injective $R$-module (by [13, Proposition 5.2.10, p. $148])$. Since $N$ is SS- $M$-injective, then we can extend $f$ to an $R$-homomorphism $\alpha: M \rightarrow N$. By projectivity of $M$, thus $\alpha$ can be lifted to an $R$-homomorphism $\tilde{\alpha}: M \rightarrow D$ such that $h \tilde{\alpha}=\alpha$. Let $\tilde{f}: K \rightarrow D$ be the restriction of $\tilde{\alpha}$ over $K$. Obviously, $h \tilde{f}=f$ and this implies that $K$ is projective.

(7) $\Rightarrow$ (1) Let $h: N \rightarrow L$ be an $R$-epimorphism, where $N$ and $L$ are right $R$-modules, and $N$ is SS-M-injective. Let $K$ be any semisimple small submodule of $M, f: K \rightarrow L$ be any $R$-homomorphism, and $i$ is the inclusion map. By hypothesis, $K$ is projective, thus $f$ can be lifted to $R$ homomorphism $g: K \rightarrow N$ such that $h g=f$. Since $N$ is SS- $M$-injective, then there exists $R$-homomorphism $\tilde{g}: M \rightarrow N$ such that $\tilde{g} i=g$. Put $\beta=h \tilde{g}: M \rightarrow L$. Thus $\beta i=h \tilde{g} i=h g=f$. Hence $L$ is an SS-Minjective right $R$-module.

(1) $\Rightarrow(4)$ Let $N_{1}$ and $N_{2}$ be two SS-M-injective submodules of a right $R$-module $N$. Then $N_{1}+N_{2}$ is a homomorphic image of the direct sum $N_{1} \oplus N_{2}$. Since $N_{1} \oplus N_{2}$ is SS-M-injective, thus $N_{1}+N_{2}$ is SS-Minjective by hypothesis.
(6) $\Rightarrow(3)$ Let $E$ be an injective right $R$-module and $N \hookrightarrow E$. Let $Q=E \oplus E, K=\{(n, n) \mid n \in N\}, \bar{Q}=$ $Q / K, H_{1}=\{y+K \in \bar{Q} \mid y \in E \oplus 0\}$ and $H_{2}=$ $\{y+K \in \bar{Q} \mid y \in 0 \oplus E\}$. Then $\bar{Q}=H_{1}+H_{2}$. Since $(E \oplus 0) \cap K=0$ and $(0 \oplus E) \cap K=0$, thus $E \cong H_{i}$, $i=1,2$. Since $H_{1} \cap H_{2}=\{y+K \in \bar{Q} \mid \quad y \in N \oplus$ $0\}=\{y+K \in \bar{Q} \mid \quad y \in 0 \bigoplus N\}$, thus $H_{1} \cap H_{2} \cong N$ under $y \mapsto y+K$ for all $y \in N \bigoplus 0$. By hypothesis, $\bar{Q}$ is SS-M-injective. Since $H_{1}$ is injective, thus $\bar{Q}=H_{1} \oplus$ $A$ for some $A \hookrightarrow \bar{Q}$, so $A \cong\left(H_{1}+H_{2}\right) / H_{1} \cong$ $H_{2} /\left(H_{1} \cap H_{2}\right) \cong E / N$. By Theorem $2.4(5), E / N$ is SS- $M$-injective.

Corollary 2.15. The following statements are equivalent for a ring $R$ :

(1) Every quotient of an SS-injective right $R$-module is SS-injective.

(2) Every quotient of a soc-injective right $R$-module is SS-injective.

(3) Every quotient of a small injective right $R$-module is SS-injective.

(4) Every quotient of an injective right $R$-module is SS-injective.

(5) Every sum of two SS-injective submodules of any right $R$-module is SS-injective.

(6) Every sum of two soc-injective submodules of any right $R$-module is SS-injective.

(7) Every sum of two small injective submodules of any right $R$-module is $\mathrm{SS}$-injective.

(8) Every sum of two injective submodules of any right $R$-module is SS-injective.

(9) Every semisimple small submodule of any projective right $R$-module is projective.

(10) Every semisimple small submodule of any finitely generated projective right $R$-module is projective.

(11) Every semisimple small submodule of $R_{R}$ is projective.

(12) Every simple small submodule of $R_{R}$ is projective.

(13) $S_{r} \cap J$ is projective.

(14) $S_{r}$ is projective ( $R$ is a right $P S$-ring).

Proof. The equivalence between (1), (2), (4), (5), (6), (8), (11), (12) and (13) is from Theorem 2.14.

$(1) \Rightarrow(3) \Rightarrow(4), \quad(5) \Rightarrow(7) \Rightarrow(8)$ and $(9) \Rightarrow(10) \Rightarrow(13)$ are clear.

(14) $\Rightarrow(9)$ By [1, Corollary 2.9].

(13) $\Rightarrow(14)$ Let $S_{r}=\left(S_{r} \cap J\right) \bigoplus A$, where $A=\bigoplus_{i \in I} S_{i}$ and $S_{i}$ is a right simple and direct summand of $R_{R}$, for all $i \in I$. Thus $A$ is projective, but $S_{r} \cap J$ is projective, so it follows that $S_{r}$ is projective. 
Theorem 2.16. If every simple singular right $R$ module is SS-injective, then $r(a) \subseteq{ }^{\oplus} R_{R}$ for every $a \in S_{r} \cap J$ and $S_{r}$ is projective.

Proof. Let $a \in S_{r} \cap J$ and let $A=R a R+r(a)$. Thus there exists $B \hookrightarrow R_{R}$ such that $A \oplus B \subseteq \subseteq^{e s s} R_{R}$. Assert that $A \oplus B \neq R_{R}$, then we find $I \subseteq^{\max } R_{R}$ such that $A \oplus B \subseteq I$, and so $I \subseteq \subseteq^{e s s} R_{R}$. By hypothesis, $R / I$ is SSinjective. Consider the map $\alpha: a R \rightarrow R / I$ is given by $\alpha(a r)=r+I$ which is well define $R$-homomorphism. Thus, there exists $c \in R$ with $1+I=c a+I$ and hence $1-c a \in I$. But $c a \in R a R \subseteq I$ which leads to $1 \in I$, a contradiction. Thus $A \oplus B=R_{R}$ and hence $R a R+$ $(r(a) \oplus B)=R$. Since $R a R \ll R_{R}$, then $r(a) \subseteq{ }^{\oplus} R_{R}$. Put $r(a)=(1-e) R$, for some $e^{2}=e \in R$, so it follows that $a x=a e x$ (because $(1-e) x \in r(a)$, and so $a(1-e) x=0)$ for all $x \in R$ and this leads to $a R=a e R$. Let $\gamma: e R \rightarrow a e R$ be defined by $\gamma(e r)=$ aer for all $r \in R$. Then $\gamma$ is a well defined $R$ epimorphism. Clearly, $\operatorname{ker}(\gamma)=\{$ er: aer $=0\}=$ $\{$ er: $\operatorname{er} \in r(a)\}=e R \cap r(a)=0$. Hence $\gamma$ is an isomorphism and so $a R$ is projective. Since $S_{r} \cap J$ is a direct sum of simple small right ideals, thus $S_{r} \cap J$ is projective and it follows from Corollary 2.15 that $S_{r}$ is projective.

Corollary 2.17. A ring $R$ is right mininjective and every singular simple right $R$-module is SS-injective if and only if $R$ is a right universally mininjective.

Proof. By Theorem 2.16 and [2, Lemma 5.1].

Recall that a ring $R$ is called zero insertive if $a R b=0$ for all $a, b \in R$ with $a b=0$ (see [3]). Note that if $R$ is zero insertive ring, then $R a R+$ $r(a) \subseteq \subseteq^{e s s} R_{R}$ for every $a \in R$ (see [3, Lemma 2.11]).

Proposition 2.18. Let $R$ be a zero insertive ring. If every simple singular right $R$-module is SS-injective, then $R$ is right universally mininjective.

Proof. Let $a \in S_{r} \cap J$. We claim that $R a R+r(a)=$ $R$, thus $r(a)=R$ (since $R a R \ll R$ ), so $a=0$ and this means that $S_{r} \cap J=0$. Otherwise, if $R a R+r(a) \subsetneq R$, then there exists a maximal right ideal $I$ of $R$ such that $R a R+r(a) \subseteq I$. Since $I \subseteq{ }^{e s s} R_{R}$ by Lemma 2.1.22, then $R / I$ is SS-injective by hypothesis. Consider $\alpha: a R \rightarrow R / I$ is given by $\alpha(a r)=r+I$ for all $r \in R$ which is well defined $R$-homomorphism. Thus $1+I=$ $c a+I$ for some $c \in R$. Since $c a \in R a R \subseteq I$, then $1 \in I$ and this contradicts the maximality of $I$, so we must have $R a R+r(a)=R$ and this ends the proof.

Theorem 2.19. If $M$ is a finitely generated right $R$ module, then the following statements are equivalent:

(1) $\operatorname{soc}(M) \cap J(M)$ is a noetherian $R$-module.

(2) $\operatorname{soc}(M) \cap J(M)$ is finitely generated.

(3) Any direct sum of SS- $M$-injective right $R$-modules is SS- $M$-injective.

(4) Any direct sum of soc- $M$-injective right $R$-modules is SS- $M$-injective.
(5) Any direct sum of injective right $R$-modules is SS$M$-injective.

(6) $K^{(S)}$ is SS-M-injective for every injective right $R$ module $K$ and for any index set $S$.

(7) $K^{(\mathbb{N})}$ is SS- $M$-injective for every injective right $R$ module $K$.

Proof. $(1) \Rightarrow(2)$ and $(3) \Rightarrow(4) \Rightarrow(5) \Rightarrow(6) \Rightarrow(7)$ Clear.

(2) $\Rightarrow$ (3) Let $E=\bigoplus_{i \in I} M_{i}$ be a direct sum of SS-Minjective right $R$-modules and $f: N \rightarrow E$ be a right $R$ homomorphism where $N$ is a semisimple small submodule of $M$. Since $\operatorname{soc}(M) \cap J(M)$ is finitely generated, thus $N$ is finitely generated and hence $f(N) \subseteq \bigoplus_{i \in I_{1}} M_{i}$, for a finite subset $I_{1}$ of $I$. Since a finite direct sums of SS- $M$-injective right $R$-modules is SS- $M$-injective, thus $\bigoplus_{i \in I_{1}} M_{i}$ is SS- $M$-injective and hence $f$ can be extended to an $R$-homomorphism $g: M \rightarrow E$. Thus $E$ is SS- $M$-injective.

(7) $\Rightarrow$ (1) Let $N_{1} \subseteq N_{2} \subseteq \cdots$ be a chain of submodules of $\operatorname{soc}(M) \cap J(M)$. For each $i \geq 1$, let $E_{i}=E\left(M / N_{i}\right)$ and $E=\oplus_{i=1}^{\infty} E_{i}$. For every $i \geq 1$, we put $M_{i}=\prod_{j=1}^{\infty} E_{j}=$ $E_{i} \oplus\left(\prod_{\substack{j \neq 1 \\ j \neq i}}^{\infty} E_{j}\right)$, then $M_{i}$ is injective. By hypothesis, $\bigoplus_{i=1}^{\infty} M_{i}=\left(\bigoplus_{i=1}^{\infty} E_{i}\right) \oplus\left(\bigoplus_{i=1}^{\infty} \prod_{\substack{j=1 \\ j \neq i}}^{\infty} E_{j}\right) \quad$ is $\quad$ SS $-M$ injective, so it follows from Theorem 2.4 (5) that $E$ is SS- $M$-injective. Define $f: U=\cup_{i=1}^{\infty} N_{i} \rightarrow E$ by $f(m)=\left(m+N_{i}\right)_{i}$. It is clear that $f$ is a well defined $R$-homomorphism. Since $M$ is finitely generated, thus $\operatorname{soc}(M) \cap J(M)$ is a semisimple small submodule of $M$ and hence $\bigcup_{i=1}^{\infty} N_{i}$ is a semisimple small submodule of $M$, so $f$ can be extended to a right $R$-homomorphism $g: M \rightarrow E$. Since $M$ is finitely generated, then we have $g(M) \subseteq \bigoplus_{i=1}^{n} E\left(M / N_{i}\right)$ for some $n$ and hence $f(U) \subseteq$ $\bigoplus_{i=1}^{n} E\left(M / N_{i}\right)$. Since $\pi_{i} f(x)=\pi_{i}\left(\left(x+N_{j}\right)_{j \geq 1}\right)=$ $x+N_{i} \quad$ for all $\quad x \in U$ and $i \geq 1$, where $\pi_{i}: \oplus_{j \geq 1} E\left(M / N_{i}\right) \rightarrow E\left(M / N_{i}\right)$ be the projection map. Thus $\pi_{i} f(U)=U / N_{i}$ for all $i \geq 1$. Since $f(U) \subseteq$ $\bigoplus_{i=1}^{n} E\left(M / N_{i}\right)$. Thus $U / N_{i}=\pi_{i} f(U)=0$, for all $i \geq n+1$, so $U=N_{i}$ for all $i \geq n+1$ and hence the chain $\quad N_{1} \subseteq N_{2} \subseteq \cdots \quad$ terminates at $N_{n+1}$. Thus $\operatorname{soc}(M) \cap J(M)$ is a noetherian $R$-module.

Corollary 2.20. If $N$ is a finitely generated right $R$ module, then the following statements are equivalent:

(1) $\operatorname{soc}(N) \cap J(N)$ is finitely generated.

(2) $M^{(S)}$ is SS- $N$-injective for every soc- $N$-injective right $R$-module $M$ and for any index set $S$.

(3) $\quad M^{(S)}$ is SS- $N$-injective for every SS- $N$-injective right $R$-module $M$ and for any index set $S$.

(4) $M^{(\mathbb{N})}$ is SS- $N$-injective for every soc- $N$-injective right $R$-module $M$.

(5) $M^{(\mathbb{N})}$ is SS- $N$-injective for every SS- $N$-injective right $R$-module $M$. 
Proof. By Theorem 2.19.

Corollary 2.21. The following statements are equivalent :

(1) $S_{r} \cap J$ is finitely generated.

(2) Any direct sum of SS-injective right $R$-modules is SS-injective.

(3) Any direct sum of soc-injective right $R$-modules is SS-injective.

(4) Any direct sum of small injective right $R$-modules is SS-injective.

(5) Any direct sum of injective right $R$-modules is ssinjective.

(6) $M^{(S)}$ is SS-injective for every injective right $R$ module $M$ and for any index set $S$.

(7) $\quad M^{(S)}$ is SS-injective for every soc-injective right $R$-module $M$ and for any index set $S$.

(8) $\quad M^{(S)}$ is SS-injective for every small injective right $R$-module $M$ and for any index set $S$.

(9) $\quad M^{(S)}$ is SS-injective for every SS-injective right $R$ module $M$ and for any index set $S$.

(10) $\quad M^{(\mathbb{N})}$ is SS-injective for every injective right $R$ module $M$.

(11) $M^{(\mathbb{N})}$ is SS-injective for every soc-injective right $R$-module $M$.

(12) $\quad M^{(\mathbb{N})}$ is SS-injective for every small injective right $R$-module $M$.

(13) $\quad M^{(\mathbb{N})}$ is SS-injective for every SS-injective right $R$ module $M$.

Proof. By applying Theorem 2.19 and Corollary 2.20.

\section{Strongly SS-Injective Modules}

Proposition 3.1. A right $R$-module $M$ is a strongly SS-injective if and only if every $R$-homomorphism $\alpha: A \rightarrow M$ extends to $N$, for all right $R$-module $N$, where $A \ll N$ and $\alpha(A)$ is a semisimple submodule in M.

\section{Proof. $(\Leftarrow)$ Clear.}

$\Leftrightarrow$ Let $A$ be a small submodule of $N$, and $\alpha: A \rightarrow M$ be an $R$-homomorphism with $\alpha(A)$ is a semisimple submodule of $M$. If $B=\operatorname{ker}(\alpha)$, then $\alpha$ induces an $R$ homomorphism $\tilde{\alpha}: A / B \rightarrow M$ defined by $\tilde{\alpha}(a+B)=$ $\alpha(a)$, for all $a \in A$. Clearly, $\tilde{\alpha}$ is well define because if $a_{1}+B=a_{2}+B$ we have $a_{1}-a_{2} \in B$, so $\alpha\left(a_{1}\right)=$ $\alpha\left(a_{2}\right)$, that is $\tilde{\alpha}\left(a_{1}+B\right)=\tilde{\alpha}\left(a_{2}+B\right)$. Since $M$ is strongly SS-injective and $A / B$ is semisimple and small in $N / B$, thus $\tilde{\alpha}$ extends to an $R$-homomorphism $\gamma: N / B \rightarrow M$. If $\pi: N \rightarrow N / B$ is the canonical map, then the $R$-homomorphism $\beta=\gamma \pi: N \rightarrow M$ is an extension of $\alpha$ such that if $a \in A$, then $\beta(a)=$ $(\gamma \pi)(a)=\gamma(a+B)=\tilde{\alpha}(a+B)=\alpha(a)$ as desired.

\section{Corollary 3.2.}

(1) Let $M$ be a semisimple right $R$-module. If $M$ is a strongly SS-injective, then $M$ is a small injective.

(2) If every simple right $R$-module is strongly SSinjective, then $R$ is a semiprimitive ring.

Proof. (1) By Proposition 3.1.

(2) By (1) and applying [3, Theorem 2.8].

Remark 3.3. The converse of Corollary 3.2 is not true ( see Example 3.8).

Theorem 3.4. If $M$ is a strongly SS-injective ( or just SS- $E(M)$-injective) right $R$-module, then for every semisimple small submodule $A$ of $M$, there is an injective $R$-module $E_{A}$ such that $M=E_{A} \oplus T_{A}$ where $T_{A} \hookrightarrow M$ with $T_{A} \cap A=0$. Moreover, if $A \neq 0$, then $E_{A}$ can be taken $A \subseteq \subseteq^{e s s} E_{A}$.

Proof. Let $A$ be a semisimple small submodule of $M$. If $A=0$, we end the proof by taking $E_{A}=0$ and $T_{A}=M$. Suppose that $A \neq 0$ and let $i_{1}, i_{2}$ and $i_{3}$ be inclusion maps and $D_{A}=E(A)$ is the injective hull of $A$ in $E(M)$. Since $M$ is strongly SS-injective, thus $M$ is SS- $E(M)$-injective. Since $A$ is a semisimple small submodule of $M$, so it follows from [8, Lemma 5.1.3 (a)] that $A$ is a semisimple small submodule in $E(M)$ and hence there exists an $R$-homomorphism $\alpha: E(M) \rightarrow M$ such that $\alpha i_{2} i_{1}=i_{3}$. Put $\beta=$ $\alpha i_{2}: D_{A} \rightarrow M$, thus $\beta$ is an extension of $i_{3}$. Since $A \subseteq{ }^{e s s} D_{A}, \beta$ is an $R$-monomorphism. Put $E_{A}=\beta\left(D_{A}\right)$. Since $E_{A}$ is an injective submodule of $M$, thus $M=$ $E_{A} \oplus T_{A}$ for some $T_{A} \hookrightarrow M$. Since $\beta(A)=A, A \subseteq$ $\beta\left(D_{A}\right)=E_{A}$ and this means that $T_{A} \cap A=0$. Moreover, define $\tilde{\beta}=\beta: D_{A} \rightarrow E_{A}$, thus $\tilde{\beta}$ is an isomorphism. Since $A \subseteq{ }^{\text {ess }} D_{A}$, thus $\tilde{\beta}(A) \subseteq{ }^{e s s} E_{A}$. But $\tilde{\beta}(A)=$ $\beta(A)=A$, so $A \subseteq^{\text {ess }} E_{A}$.

Corollary 3.5. If $M$ is a right $R$-module has a semisimple small submodule $A$ such that $A \subseteq \subseteq^{e s s} M$, then the following statements are equivalent:

(1) $M$ is injective.

(2) $M$ is strongly SS-injective.

(3) $M$ is SS- $E(M)$-injective.

Proof. (1) $\Rightarrow(2)$ and $(2) \Rightarrow(3)$ are obvious.

(3) $\Rightarrow$ (1) By Theorem 3.4, we can write $M=E_{A} \oplus T_{A}$ where $E_{A}$ injective and $T_{A} \cap A=0$. Since $A \subseteq{ }^{e s s} M$, thus $T_{A}=0$ and hence $M=E_{A}$. Therefore $M$ is an injective $R$-module.

Example 3.6. $\mathbb{Z}_{4}$ as $\mathbb{Z}$-module is not strongly SSinjective. In particular, $\mathbb{Z}_{4}$ is not $S S-\mathbb{Z}_{2} \infty$-injective.

Proof. Assume that $\mathbb{Z}_{4}$ is strongly SS-injective $\mathbb{Z}$ module. Let $A=<\overline{2}>=\{\overline{0}, \overline{2}\}$. It is clear that $A$ is a semisimple small and essential submodule of $\mathbb{Z}_{4}$ as $\mathbb{Z}$ module. By Corollary $3.5, \mathbb{Z}_{4}$ is injective $\mathbb{Z}$-module and this a contradiction. Thus $\mathbb{Z}_{4}$ as $\mathbb{Z}$-module is not strongly SS-injective. Moreover, Since $E\left(\mathbb{Z}_{2^{2}}\right)=\mathbb{Z}_{2}$ 为 as $\mathbb{Z}$-module, thus $\mathbb{Z}_{4}$ is not $\mathrm{SS}-\mathbb{Z}_{2} \infty$-injective, by Corollary 3.5. 
Corollary 3.7. Let $M$ be a right $R$-module such that $\operatorname{soc}(M) \cap J(M) \ll M$ (in particular, if $M$ is finitely generated). If $M$ is strongly SS-injective, then $M=$ $E \oplus T$, where $E$ is injective and $T \cap \operatorname{soc}(M) \cap J(M)=$ 0 . Moreover, if $\operatorname{soc}(M) \cap J(M) \neq 0$, then we can take $\operatorname{soc}(M) \cap J(M) \subseteq \subseteq^{\text {ess }} E$.

Proof. By taking $A=\operatorname{soc}(M) \cap J(M)$ and applying Theorem 3.4.

The following example shows that the converse of Theorem 3.4 and Corollary 3.7 is not true.

Example 3.8. Let $M=\mathbb{Z}_{6}$ as $\mathbb{Z}$-module. Since $J(M)=0$ and $\operatorname{soc}(M)=M$, thus $\operatorname{soc}(M) \cap J(M)=0$. So, we can write $M=0 \oplus M$ with $M \cap(\operatorname{soc}(M) \cap$ $J(M))=0$. Let $N=\mathbb{Z}_{8}$ as $\mathbb{Z}$-module. Since $J(N)=<$ $\overline{2}>$ and $\operatorname{soc}(N)=<\overline{4}>$. Define $\gamma: \operatorname{soc}(N) \cap J(N) \rightarrow$ $M$ by $\gamma(\overline{4})=\overline{3}$, thus $\gamma$ is a $\mathbb{Z}$-homomorphism. Assume that $M$ is strongly SS-injective, thus $M$ is SS- $N$ injective, so there exists $\mathbb{Z}$-homomorphism $\beta: N \rightarrow M$ such that $\beta \circ i=\gamma$, where $i$ is the inclusion map from $\operatorname{soc}(N) \cap J(N)$ to $N$. Since $\beta(J(N)) \subseteq J(M)$, thus $\overline{3}=\gamma(\overline{4})=\beta(\overline{4}) \in \beta(J(N)) \subseteq J(M)=0$ and this contradiction, so $M$ is not strongly SS-injective $\mathbb{Z}$ module.

Corollary 3.9. The following statements are equivalent:

(1) $\operatorname{soc}(M) \cap J(M)=0$, for all right $R$-module $M$.

(2) Every right $R$-module is strongly SS-injective.

(3) Every simple right $R$-module is strongly SSinjective.

Proof. By Proposition 2.11.

Lemma 3.10. Let $M$ and $C$ be right $R$-modules and $N \hookrightarrow M$ with $M / N$ is a semisimple. Then every $R$ homomorphism from a submodule (resp. semisimple submodule) $A$ of $M$ to $C$ can be extended to an $R$ homomorphism from $M$ to $C$ if and only if every $R$ homomorphism from a submodule (resp. semisimple submodule) $B$ of $N$ to $C$ can be extended to an $R$ homomorphism from $M$ to $C$.

Proof. $(\Rightarrow)$ is obtained directly.

$(\Leftarrow)$ let $f$ be an $R$-homomorphism from a submodule $A$ of $M$ to $C$. Since $M / N$ is a semisimple, there exists $L \hookrightarrow M$ such that $A+L=M$ and $A \cap L \subseteq N$ ( see [6, Proposition 2.1]). Thus there exists an $R$ homomorphism $g: M \rightarrow C$ such that $g(x)=f(x)$ for all $x \in A \cap L$. Define $h: M \rightarrow C$ such that for any $x=a+\ell, a \in A, \ell \in L, h(x)=f(a)+g(\ell)$. Thus $h$ is a well define $R$-homomorphism, because if $a_{1}+\ell_{1}=$ $a_{2}+\ell_{2}, a_{i} \in A, \ell_{i} \in L, i=1,2$, then $a_{1}-a_{2}=\ell_{2}-$ $\ell_{1} \in A \cap L$, that is $f\left(a_{1}-a_{2}\right)=g\left(\ell_{2}-\ell_{1}\right)$ which leads to $h\left(a_{1}+\ell_{1}\right)=h\left(a_{2}+\ell_{2}\right)$. Therefore $h$ is a well define $R$-homomorphism and extension of $f$.
Corollary 3.11. For right $R$-modules $M$ and $N$, the following hold:

(1) If $M$ is finitely generated and $M / J(M)$ is semisimple right $R$-module, then $N$ is soc- $M$ injective if and only if $N$ is SS- $M$-injective.

(2) If $M / \operatorname{soc}(M)$ is semisimple right $R$-module, then $N$ is soc- $M$-injective if and only if $N$ is $M$-injective.

(3) If $R / S_{r}$ is semisimple as right $R$-module, then $N$ is soc-injective if and only if $N$ is injective.

(4) If $R / S_{r}$ is semisimple as right $R$-module, then $N$ is SS-injective if and only if $N$ is small injective.

Proof. $(1)(\Rightarrow)$ Clear.

$(\Leftarrow)$ Since $N$ is a right SS- $M$-injective, thus every $R$ homomorphism from a semisimple small submodule of $M$ to $N$ extends to $M$. Since $M$ is finitely generated, thus $J(M) \ll M$ and hence every $R$-homomorphism from any semisimple submodule of $J(M)$ to $N$ extends to $M$. Since $M / J(M)$ is semisimple, thus every $R$ homomorphism from any semisimple submodule of $M$ to $N$ extends to $M$ by Lemma 3.10. Therefore, $N$ is soc$M$-injective right $R$-module.

(2) $(\Rightarrow)$ Since $N$ is soc- $M$-injective. Thus every $R$ homomorphism from any submodule of $\operatorname{soc}(M)$ to $N$ extends to $M$. Since $M / \operatorname{soc}(M)$ is semisimple, thus Lemma 3.10 implies that every $R$-homomorphism from any submodule of $M$ to $N$ extends to $M$. Hence $N$ is $M$ injective.

$(\Leftarrow)$ Clear.

(3) By (2).

(4) Since $R / S_{r}$ is semisimple as right $R$-module, thus $J\left(R / S_{r}\right)=0$. By [8, Theorem 9.1.4(b)], we have $J \subseteq S_{r}$ and hence $J=J \cap S_{r}$. Thus $N$ is SS-injective if and only is $N$ is small injective.

Corollary 3.12. Let $R$ be a semilocal ring, then $S_{r} \cap J$ is finitely generated if and only if $S_{r}$ is finitely generated.

Proof. Suppose that $S_{r} \cap J$ is finitely generated. By Corollary 2.21, every direct sum of soc-injective right $R$-modules is SS-injective. Thus it follows from Corollary 3.11 (1) and [1, Corollary 2.11] that $S_{r}$ is finitely generated.

Theorem 3.13. If $R$ is a right perfect ring, then $M$ is a strongly soc-injective right $R$-module if and only if $M$ is a strongly SS-injective.

Proof. $(\Rightarrow)$ Clear.

$(\Leftarrow)$ Let $R$ be a right perfect ring and $M$ be a strongly SS-injective right $R$-module. Since $R$ is a semilocal ring, thus it follows from [14, Theorem 3.5] that every right $R$-module $N$ is semilocal and hence $N / J(N)$ is semisimple right $R$-module. Since $R$ is a right perfect ring, the Jacobson radical of every right $R$-module is small by [13, Theorem 4.3 and 4.4, p. 69]. Thus $N / J(N)$ is semisimple and $J(N) \ll N$, for any $N \in$ Mod- $R$. Since $M$ is strongly SS-injective it follows Lemma 3.10 implies that $M$ is strongly soc-injective. 
Corollary 3.14. A ring $R$ is $Q F$ if and only if every strongly SS-injective right $R$-module is projective.

Proof. $(\Rightarrow)$ If $R$ is $Q F$ ring, then $R$ is a right perfect ring, so by Theorem 3.13 and [1, Proposition 3.7] we have that every strongly SS-injective right $R$-module is projective.

$(\Leftarrow)$ By hypothesis we have that every injective right $R$ module is projective and hence $R$ is $Q F$ ring ( see for instance [11, Proposition 12.5.13]).

Theorem 3.15. The following statements are equivalent for a ring $R$ :

(1) Every direct sum of strongly SS-injective right $R$ modules is injective.

(2) Every direct sum of strongly soc-injective right $R$ modules is injective.

(3) $R$ is right artinian.

Proof. (1) $\Rightarrow(2)$ Clear.

(2) $\Rightarrow$ (3) Since every direct sum of strongly soc-injective right $R$-modules is injective. Thus $R$ is right noetherian and right semiartinian by $[1$, Theorem 3.3 and Theorem 3.6], so it follows from [15, Proposition VIII.5.2, p. 189] that $R$ is right artinian.

(3) $\Rightarrow(1)$ By hypothesis, $R$ is right perfect and right noetherian. It follows from Theorem 3.13 and [1, Theorem 3.3] that every direct sum of strongly SSinjective right $R$-modules is strongly soc-injective. Since $R$ is right semiartinian, so [1, Theorem 3.6] implies that every direct sum of strongly SS-injective right $R$-modules is injective.

Recall that a submodule $K$ of a right $R$-module $M$ is called $t$-essential in $M$ (written $K \subseteq \subseteq^{\text {tes }} M$ ) if for every submodule $L$ of $M, K \cap L \subseteq Z_{2}(M)$ implies that $L \subseteq Z_{2}(M)$ (see [16]). A right $R$-module $M$ is said to be $t$-semisimple if every submodule $A$ of $M$ there exists a direct summand $B$ of $M$ such that $B \subseteq^{\text {tes }} A$ (see [16]). A ring $R$ is said to be right $V$-ring ( $G V$-ring, $S I$-ring, respectively) if every simple (simple singular, singular, respectively) right $R$-module is injective. A right $R$ module is called strongly s-injective if every $R$ homomorphism from $K$ to $M$ extends to $N$ for every right $R$-module $N$, where $K \subseteq Z(N)$ (see [17]). In the next results, we will give the connection between injectivity and strongly s-injectivity and we characterize $V$-rings, $G V$-rings, $S I$-rings and semisimple rings by this connection.

Theorem 3.16. If $R$ is a right $t$-semisimple, then a right $R$-module $M$ is injective if and only if $M$ is strongly s-injective.

Proof. $(\Rightarrow)$ Obvious.

$(\Leftrightarrow)$ Let $M$ be a strongly s-injective, $Z_{2}(M)$ is injective by [17, Proposition 3, p. 27]. Thus every $R$ homomorphism $f: K \rightarrow M$, where $K \subseteq Z_{2}^{r}$ extends to $R$ by [17, Lemma 1 , p. 26]. Since $R$ is a right $t$ semisimple, thus $R / Z_{2}^{r}$ is a right semisimple by [16,
Theorem 2.3]. So by applying Lemma 3.10, we conclude that $M$ is injective.

Corollary 3.17. A ring $R$ is right $S I$ and right $t$ semisimple if and only if it is semisimple.

Proof. $(\Rightarrow)$ Since $R$ is a right $S I$-ring, thus every right $R$-module is strongly s-injective by [17, Theorem $1, \mathrm{p}$. $29]$. By Theorem 3.16, we have that every right $R$ module is injective and hence $R$ is semisimple ring.

$(\Leftarrow)$ Clear.

Corollary 3.18. If $R$ is a right $t$-semisimple ring. Then $R$ is right $V$-ring if and only if $R$ is right $G V$-ring.

Proof. By [17, Proposition 5, p. 28] and Theorem 3.16.

Corollary 3.19. If $R$ is a right $t$-semisimple ring, then $R / S_{r}$ is noetherian right $R$-module if and only if $R$ is right noetherian.

Proof. If $R / S_{r}$ is noetherian right $R$-module, then every direct sum of injective right $R$-modules is strongly s-injective by [17, Proposition 6]. Since $R$ is right $t$-semisimple, so it follows from Theorem 3.16 that every direct sum of injective right $R$-modules is injective and hence $R$ is right noetherian. The converse is clear.

\section{SS-Injective Rings}

We recall that the dual of a right $R$-module $M$ is $M^{d}=\operatorname{Hom}_{R}\left(M, R_{R}\right)$ and clearly that $M^{d}$ is a left $R$ module.

Proposition 4.1. The following statements are equivalent for a ring $R$ :

(1) $R$ is a right $\mathrm{SS}$-injective ring.

(2) If $K$ is a semisimple right $R$-module, $P$ and $Q$ are finitely generated projective right $R$-modules, $\beta: K \rightarrow P$ is an $R$-monomorphism with $\beta(K) \ll P$ and $f: K \rightarrow Q$ is an $R$-homomorphism, then $f$ can be extended to an $R$-homomorphism $h: P \rightarrow Q$.

(3) If $M$ be a right semisimple $R$-module and $f$ is a nonzero $R$-monomorphism from $M$ to $R_{R}$ with $f(M) \ll R_{R}$, then $M^{d}=R f$.

Proof. (2) $\Rightarrow(1)$ Clear.

(1) $\Rightarrow(2)$ Since $Q$ finitely generated, there is an $R$ epimorphism $\alpha_{1}: R^{n} \rightarrow Q$ for some $n \in \mathbb{Z}^{+}$. Since $Q$ is a projective, there is an $R$-homomorphism $\alpha_{2}: Q \rightarrow R^{n}$ such that $\alpha_{1} \alpha_{2}=I_{Q}$. Define $\tilde{\beta}: K \rightarrow \beta(K)$ by $\tilde{\beta}(a)=$ $\beta(a)$ for all $a \in K$. Since $R$ is a right SS-injective ring by hypothesis, it follows from Proposition 2.8 and Corollary 2.5 (1) that $R^{n}$ is a right SS- $P$-injective $R$ module. So there exists an $R$-homomorphism $h: P \rightarrow$ $R^{n}$ such that $h i=\alpha_{2} f \tilde{\beta}^{-1}$. Put $g=\alpha_{1} h: P \rightarrow Q$. Thus $g i=\left(\alpha_{1} h\right) i=\alpha_{1}\left(\alpha_{2} f \tilde{\beta}^{-1}\right)=f \tilde{\beta}^{-1}$ and hence $(g \beta)(a)=g(i(\beta(a)))=\left(f \tilde{\beta}^{-1}\right)(\beta(a))=f(a)$ for all $a \in K$. Therefore, there is an $R$-homomorphism $g: P \rightarrow Q$ such that $g \beta=f$. 
(1) $\Rightarrow(3)$ Let $g \in M^{d}$, we have $g f^{-1}: f(M) \rightarrow R_{R}$, since $f(M)$ is a semisimple small right ideal of $R$ and $R$ is a right SS-injective ring ( by hypothesis), $g f^{-1}=a$. for some $a \in R$. Therefore, $g=a f$ and hence $M^{d}=$ $R f$.

(3) $\Rightarrow$ (1) Let $f: K \rightarrow R$ be a right $R$-homomorphism, where $K$ is a semisimple small right ideal of $R$ and $i: K \rightarrow R$ be the inclusion map, thus by (3) we have $K^{d}=R i$ and hence $f=c i$ in $K^{d}$ for some $c \in R$. Thus there is $c \in R$ such that $f(a)=c a$ for all $a \in K$ and this implies that $R$ is a right SS-injective ring.

\section{Example 4.2.}

(1) Every universally mininjective ring is SS-injective, but not conversely (see Example 5.6).

(2) The two classes of universally mininjective rings and soc-injective rings are different ( see Example 5.6 and Example 5.7).

Lemma 4.3. Let $R$ be a right SS-injective ring. Then:

(1) $R$ is a right mininjective ring.

(2) $\operatorname{lr}(a)=R a$ for all $a \in S_{r} \cap J$.

(3) $r(a) \subseteq r(b), a \in S_{r} \cap J, b \in R$ implies $R b \subseteq R a$.

(4) $l(b R \cap r(a))=l(b)+R a$, for all $a \in S_{r} \cap J$, $b \in R$.

(5) $l\left(K_{1} \cap K_{2}\right)=l\left(K_{1}\right)+l\left(K_{2}\right)$, for all semisimple small right ideals $K_{1}$ and $K_{2}$ of $R$.

\section{Proof. Clear.}

The following is an example of a right mininjective ring which is not right SS-injective.

Example 4.4. (The Björk Example [5, Example 2.5, p. 38]). Let $F$ be a field and let $a \mapsto \bar{a}$ be an isomorphism $F \rightarrow \bar{F} \subseteq F$, where the subfield $\bar{F} \neq F$. Let $R$ denote the left vector space on basis $\{1, t\}$, and make $R$ into an $F$-algebra by defining $t^{2}=0$ and $t a=\bar{a} t$ for all $a \in F$. By [5, Example 2.5 and 5.2, p. 38 and 97] we have $R$ is a right principally injective and local ring. It is mentioned in [1, Example 4.15], that $R$ is not right soc-injective. Since $R$ is local, thus by Corollary 3.11 (1), $R$ is not right SS-injective ring.

Proposition 4.5. Let $R$ be a right SS-injective ring. Then :

(1) If $R a$ is a simple left ideal of $R$, then $\operatorname{soc}(a R) \cap$ $J(a R)$ is zero or simple.

(2) $\quad r l\left(S_{r} \cap J\right)=S_{r} \cap J$ if and only if $r l(N)=N$ for all semisimple small right ideals $N$ of $R$.

Proof. (1) Suppose that $\operatorname{soc}(a R) \cap J(a R)$ is a nonzero. Let $x_{1} R$ and $x_{2} R$ be any simple small right ideals of $R$ with $x_{i} \in a R, i=1,2$. If $x_{1} R \cap x_{2} R=0$, then by Lemma $4.3(5), l\left(x_{1}\right)+l\left(x_{2}\right)=R$. Since $x_{i} \in a R$, thus $x_{i}=a r_{i}$ for some $r_{i} \in R, i=1,2$, that is $l(a) \subseteq l\left(a r_{i}\right)=l\left(x_{i}\right), i=1,2$. Since $R a$ is a simple, then $l(a) \subseteq^{\max } R$, that is $l\left(x_{1}\right)=l\left(x_{2}\right)=l(a)$.
Therefore, $l(a)=R$ and hence $a=0$ and this contradicts the minimality of $R a$. Thus $\operatorname{soc}(a R) \cap$ $J(a R)$ is simple.

(2) Suppose that $r l\left(S_{r} \cap J\right)=S_{r} \cap J$ and let $N$ be a semisimple small right ideal of $R$, trivially we have $N \subseteq r l(N)$. If $N \cap x R=0$ for some $x \in \operatorname{rl}(N)$, then by Lemma $4.3(5), l(N \cap x R)=l(N)+l(x R)=R$, since $x \in r l(N) \subseteq r l\left(S_{r} \cap J\right)=S_{r} \cap J$. If $y \in l(N)$, then $y x=0$, that is $y(x r)=0$ for all $r \in R$ and hence $l(N) \subseteq l(x R)$. Thus $l(x R)=R$, so $x=0$ and this means that $N \subseteq \subseteq^{e s s} r l(N)$. Since $N \subseteq \subseteq^{e s s} r l(N) \subseteq$ $r l\left(S_{r} \cap J\right)=S_{r} \cap J$, it follows that $N=\operatorname{rl}(N)$. The converse is trivial.

Recall that a right ideal $I$ of $R$ is said to be lie over summand of $R_{R}$, if there exists a direct decomposition $R_{R}=A_{R} \oplus B_{R}$ with $A \subseteq I$ and $B \cap I \ll R_{R}$ (see [18]) which leads to $I=A \oplus(B \cap I)$.

Lemma 4.6. Let $K$ be an $m$-generated semisimple right ideal lies over summand of $R_{R}$. If $R$ is a right SSinjective ring, then every $R$-homomorphism from $K$ to $R_{R}$ can be extended to an endomorphism of $R_{R}$.

Proof. Let $\alpha: K \rightarrow R$ be a right $R$-homomorphism. By hypothesis, $K=e R \oplus B$, for some $e^{2}=e \in R$, where $B$ is an $m$-generated semisimple small right ideal of $R$. Now, we need to prove that $K=e R \oplus(1-e) B$. Clearly, $e R+(1-e) B$ is a direct sum. Let $x \in K$, then $x=a+b$, for some $a \in e R, b \in B$, so we can write $x=a+e b+(1-e) b$ and this implies that $x \in e R \oplus$ $(1-e) B$. Conversely, let $x \in e R \oplus(1-e) B$. Thus $x=a+(1-e) b$, for some $a \in e R, b \in B$. We obtain $x=a+(1-e) b=(a-e b)+b \in e R \oplus B$. It is obvious that $(1-e) B$ is an $m$-generated semisimple small right ideal. Since $R$ is a right SS-injective, then there exists $\gamma \in \operatorname{End}\left(R_{R}\right)$ such that $\gamma_{\mid(1-e) B}=\alpha_{\mid(1-e) B}$. Define $\beta: R_{R} \rightarrow R_{R}$ by $\beta(x)=\alpha(e x)+\gamma((1-e) x)$, for all $x \in R$ which is well defined $R$-homomorphism. If $x \in K$, then $x=a+b$ where $a \in e R$ and $b \in$ $(1-e) B$, so $\beta(x)=\alpha(e x)+\gamma((1-e) x)=\alpha(a)+$ $\gamma(b)=\alpha(a)+\alpha(b)=\alpha(x)$ which yields $\beta$ is an extension of $\alpha$.

Corollary 4.7. Let $S_{r}$ be a finitely generated and lies over summand of $R_{R}$. Then $R$ is a right SS-injective ring if and only if $R$ is a right soc-injective .

Proof. By Lemma 4.6.

Recall that a ring $R$ is called right minannihilator if $r l(K)=K$ for every simple right ideal $K$ of $R$ (see [2]) (equivalently, for every simple small right ideal $K$ of $R$ ).

Corollary 4.8. For a right SS-injective ring $R$, the following hold:

(1) If $r l\left(S_{r} \cap J\right)=S_{r} \cap J$, then $R$ is right minannihilator.

(2) If $S_{\ell} \subseteq S_{r}$, then:

(a) $S_{\ell}=S_{r}$.

(b) $R$ is a left minannihilator ring. 
Proof. (1) By Proposition 4.5 (2).

(2) (a) By [2, Proposition 1.14 (4)].

(b) By Lemma 4.3 (2).

Proposition 4.9. The following statements are equivalent for a right SS-injective ring $R$ :

(1) $S_{\ell} \subseteq S_{r}$.

(2) $S_{\ell}=S_{r}$.

(3) $R$ is a left mininjective ring.

Proof. (1) $\Rightarrow$ (2) By Corollary 4.8 (2) (a).

(2) $\Rightarrow$ (3) By Corollary 4.8 (2) and [5, Corollary 2.34, p. 53], we must show that $R$ is right minannihilator ring. Let $a R$ be a simple small right ideal, then $R a$ is a simple small left ideal by [2, Theorem 1.14]. Let $0 \neq x \in$ $r l(a R)$, then $l(a) \subseteq l(x)$. Since $l(a) \subseteq \subseteq^{\max } R$, thus $l(a)=l(x)$ and hence $R x$ is simple left ideal, that is $x \in S_{r}$. Now, if $R x=R e$ for some $e^{2}=e \in R$, then $e=r x$ for some $0 \neq r \in R$. Since $(e-1) e=0$, then $(e-1) r x=0$, that is $(e-1) r a=0$ and this implies that $r a \in e R$. Thus $r a R \subseteq e R$, but $e R$ is semisimple right ideal, so $r a R \subseteq \subseteq^{\oplus} R$ and hence $r a=0$. Therefore, $r x=0$, that is $e=0$, a contradiction. Thus $x \in J$ and hence $x \in S_{r} \cap J$. Therefore, $a R \subseteq \operatorname{rl}(a R) \subseteq S_{r} \cap J$. Now, let $a R \cap y R=0$ for some $y \in \operatorname{rl}(a R)$, thus $l(a R)+l(y R)=l(a R \cap y R)=R$. Since $y \in \operatorname{rl}(a R)$, thus $l(a R) \subseteq l(y R)$ and hence $l(y R)=R$, that is $y=$ 0 . Therefore, $a R \subseteq \subseteq^{e s s} r l(a R)$, so $a R=\operatorname{rl}(a R)$ as desired.

(3) $\Rightarrow$ (1) Follows from [5, Corollary 2.34, p. 53].

Recall that a ring $R$ is said to be right minfull if it is semiperfect, right mininjective and $\operatorname{soc}(e R) \neq 0$ for each local idempotent $e \in R$ (see [5]). A ring $R$ is called right $\min -P F$, if it is a semiperfect, right mininjective, $S_{r} \subseteq{ }^{e s s} R_{R}, \operatorname{lr}(K)=K$ for every simple left ideal $K \subseteq e R$ for some local idempotent $e \in R$ (see [5]).

Corollary 4.10. Let $R$ be a right SS-injective ring, semiperfect with $S_{r} \subseteq{ }^{e s s} R_{R}$. Then $R$ is a right minfull ring and the following statements hold:

(1) Every simple right ideal of $R$ is essential in a summand.

(2) $\operatorname{soc}(e R)$ is simple and essential in $e R$ for every local idempotent $e \in R$. Moreover, $R$ is right finitely cogenerated.

(3) For every semisimple right ideal $I$ of $R$, there exists $e^{2}=e \in R$ such that $I \subseteq \subseteq^{e s s} r l(I) \subseteq{ }^{e s s} e R$.

(4) $S_{r} \subseteq S_{\ell} \subseteq \operatorname{rl}\left(S_{r}\right)$.

(5) If $I$ is a semisimple right ideal of $R$ and $a R$ is a simple right ideal of $R$ with $I \cap a R=0$, then $r l(I \oplus a R)=r l(I) \oplus \operatorname{rl}(a R)$.

(6) $\quad r l\left(\bigoplus_{i=1}^{n} a_{i} R\right)=\bigoplus_{i=1}^{n} r l\left(a_{i} R\right)$, where $\bigoplus_{i=1}^{n} a_{i} R$ is a direct sum of simple right ideals.

(7) The following statements are equivalent:

(a) $S_{r}=\operatorname{rl}\left(S_{r}\right)$.

(b) $K=r l(K)$, for every semisimple right ideals $K$ of $R$. (c) $k R=r l(k R)$, for every simple right ideals $k R$ of $R$.

(d) $S_{r}=S_{\ell}$.

(e) $\operatorname{soc}(R e)$ is a simple for all local idempotent $e \in R$.

(f) $\operatorname{soc}(R e)=S_{r} e$, for all local idempotent $e \in R$.

(g) $R$ is a left mininjective.

(h) $L=\operatorname{lr}(L)$, for every semisimple left ideals $L$ of $R$.

(i) $R$ is a left minfull ring.

(j) $S_{r} \cap J=r l\left(S_{r} \cap J\right)$.

(k) $K=r l(K)$, for every semisimple small right ideals $K$ of $R$.

(1) $L=\operatorname{lr}(L)$, for every semisimple small left ideals $L$ of $R$.

(8) If $R$ satisfies any condition of (7), then $r\left(S_{\ell} \cap\right.$ $J) \subseteq{ }^{e s s} R_{R}$.

Proof. (1), (2), (3), (4), (5) and (6) are obtained by Corollary 2.1.32 (1) and [1, Theorem 4.12].

(7) The equivalence of (a), (b), (c), (d), (e), (f), (g), (h) and (i) follows from Corollary 3.11 and [1, Theorem 4.12].

(b) $\Rightarrow$ (j) Clear.

$(\mathrm{j}) \Leftrightarrow(\mathrm{k})$ By Proposition 4.5 (2).

$(\mathrm{k}) \Rightarrow$ (c) By Corollary 4.8 (1).

(h) $\Rightarrow$ (l) Clear.

(l) $\Rightarrow$ (d) Let $R a$ be a simple left ideal of $R$. By hypothesis, $\operatorname{lr}(A)=A$ for any simple small left ideal $A$ of $R$. Since $\operatorname{lr}(A)=A$, for any simple left ideal $A$ of $R$, $\operatorname{lr}(R a)=R a$. Thus $R$ is a right min- $P F$ ring and it follows from [2, Theorem 3.14] that $S_{r}=S_{\ell}$.

(8) Let $K$ be a right ideal of $R$ such that $r\left(S_{\ell} \cap J\right) \cap$ $K=0$. Then $K r\left(S_{\ell} \cap J\right)=0$ and we have $K \subseteq$ $\operatorname{lr}\left(S_{\ell} \cap J\right)=S_{\ell} \cap J=S_{r} \cap J . \quad$ Now, $\quad r\left(\left(S_{\ell} \cap J\right)+\right.$ $l(K))=r\left(S_{\ell} \cap J\right) \cap K=0$. Since $R$ is left Kasch, then $\left(S_{\ell} \cap J\right)+l(K)=R$ by [9, Corollary 8.28 (5), p. 281]. Thus $l(K)=R$ and hence $K=0$, so $r\left(S_{\ell} \cap J\right) \subseteq{ }^{e s s} R_{R}$.

N. Zeyada, S. Hussein and A. Amin [19] introduced the notion almost-injective, a right $R$-module $M$ is called almost-injective if $M=E \oplus K$, where $E$ is injective and $K$ has zero radical. They proved that, every almost-injective right $R$-module is an injective if and only if every almost-injective is a quasi-continuous if and only if $R$ is a semilocal ring ( see [19, Theorem 2.12]). After reflect of [19, Theorem 2.12] we found it is not true always and the reason is due to the $R$ homomorphism $h:(L+J) / J \rightarrow K$ in the proof of the part of the Theorem 2.12 in [19] is not well define, so most of the other results in [19] are not necessary to be correct, because they are based on [19, Theorem2.12]. The following examples show that the contradiction in $[19$, Theorem 2.12] is exist. 
Example 4.11. In particular from the proof of the part (3) $\Rightarrow(1)$ in [19, Theorem 2.12], we consider $R=\mathbb{Z}_{8}$ and $M=K=<\overline{4}>$. Thus $M=E \oplus K$, where $E=0$ is a trivial injective $R$-module and $J(K)=0$. Let $f: L \rightarrow K$ is the identity map, where $L=K$. So, the map homomorphism $h:(L+J) / J \rightarrow K$ which is given by $h(\ell+J)=f(\ell)$ is not well define, because $J=\overline{4}+J$ but $h(J)=f(\overline{0})=\overline{0} \neq \overline{4}=f(\overline{4})=h(\overline{4}+$ $J)$.

\section{Example 4.12.}

(1) Let $R$ be an artinian ring. Assume that $R$ is not semisimple ring, then $R$ is not right $V$-ring. Thus there is simple right $R$-module is not injective. Therefore, there is almost-injective right $R$-module is not injective. So it follows from [19, Theorem 2.12] that $R$ is not semilocal. Hence, $R$ is not right artinian and this a contradiction. Thus every right artinian ring is semisimple, but this is not true in general (see below example).

(2) The ring $\mathbb{Z}_{8}$ is semilocal. Since $<\overline{4}>=\{\overline{0}, \overline{4}\}$ is almost-injective as $\mathbb{Z}_{8}$-module, then $\langle\overline{4}\rangle$ is injective $\mathbb{Z}_{8}$-module by [19, Theorem 2.12]. Thus $<\overline{4}>\subseteq^{\oplus} \mathbb{Z}_{8}$ and this a contradiction.

Theorem 4.13. The following statements are equivalent for a ring $R$ :

(1) $R$ is a semiprimitive and every almost-injective right $R$-module is quasi-continuous.

(2) $R$ is a right SS-injective and right minannihilator ring, $J$ is a right artinian, and every almost-injective right $R$-module is quasi-continuous.

(3) $R$ is a semisimple ring.

Proof. (1) $\Rightarrow(2)$ and (3) $\Rightarrow(1)$ are clear.

(2) $\Rightarrow$ (3) Let $M$ be a right $R$-module with zero Jacobson radical and let $K$ be a nonzero submodule of $M$. Thus $K \oplus M$ is a quasi-continuous. By [20, Corollary 2.14, p. 23], $K$ is an $M$-injective. Thus $K \subseteq^{\oplus} M$ and hence $M$ is semisimple. In particular, $R / J$ is a semisimple $R$ module and hence $R / J$ is artinian by [8, Theorem 9.2.2 (b), p. 219], so $R$ is semilocal ring. Since $J$ is a right artinian, then $R$ is a right artinian. So, it follows from Corollary 4.10 (7) that $R$ is right and left mininjective. Thus [2, Corollary 4.8] implies that $R$ is $Q F$ ring. By hypothesis $R \oplus(R / J)$ is quasi-continuous ( since $R$ is self-injective), so again by [20, Corollary 2.14, p. 23] we have that $R / J$ is an injective. Since $R$ is $Q F$ ring, then $R / J$ is a projective (see [8, Theorem 13.6.1]). Thus the canonical map $\pi: R \rightarrow R / J$ is a splits and hence $J \subseteq \subseteq^{\oplus} R$, that is $J=0$. Therefore, $R$ is semisimple.

\section{Strongly SS-Injective Rings}

A ring $R$ is called a right Ikeda-Nakayama ring if $l(A \cap B)=l(A)+l(B)$ for all right ideals $A$ and $B$ of $R$ (see [5, p. 148]). In the next proposition, the strongly SS-injectivity gives a new version of Ikeda-Nakayama rings.

Proposition 5.1. Let $R$ be a strongly right SSinjective ring, then $l(N \cap K)=l(N)+l(K)$ for all semisimple small right ideals $N$ and all right ideals $K$ of $R$.

Proof. Suppose that $x \in l(N \cap K)$ and define $\alpha: N+K \rightarrow R_{R}$ by $\alpha(a+b)=x a$ for all $a \in N$ and $b \in K$. Clearly, $\alpha$ is well define, because if $a_{1}+b_{1}=$ $a_{2}+b_{2}$, then $a_{1}-a_{2}=b_{2}-b_{1}$, that is $x\left(a_{1}-a_{2}\right)=$ 0 , so $\alpha\left(a_{1}+b_{1}\right)=\alpha\left(a_{2}+b_{2}\right)$. Define the $R$ homomorphism $\tilde{\alpha}:(N+K) / K \rightarrow R_{R}$ by $\tilde{\alpha}(a+K)=$ $x a$ for all $a \in N$ which induced by $\alpha$. Since $(N+K) / K \subseteq \operatorname{soc}(R / K) \cap J(R / K)$ and $R$ is a strongly right SS-injective, $\tilde{\alpha}$ can be extended to an $R$ homomorphism $\gamma: R / K \rightarrow R_{R}$. If $\gamma(1+K)=y$, for some $y \in R$, then $y(a+b)=x a$, for all $a \in N$ and $b \in K$. In particular, $y a=x a$ for all $a \in N$ and $y b=0$ for all $b \in K$. Hence $x=(x-y)+y \in l(N)+l(K)$. Therefore, $l(N \cap K) \subseteq l(N)+l(K)$. Since the converse is always holds, thus the proof is complete.

Recall that a ring $R$ is said to be right simple $J$ injective if for any small right ideal $I$ and any $R$ homomorphism $\alpha: I \rightarrow R_{R}$ with simple image, $\alpha=c$. for some $c \in R$ (see [14]).

Corollary 5.2. Every strongly right SS-injective ring is a right simple $J$-injective.

Proof. By Proposition 3.1.

Remark 5.3. The converse of Corollary 5.2 is not true (see Example 5.6).

Proposition 5.4. Let $R$ be a right Kasch and strongly right SS-injective. Then:

(1) $\operatorname{rl}(K)=K$, for every small right ideal $K$ of $R$. Moreover, $R$ is right minannihilator.

(2) If $R$ is left Kasch, then $r(J) \subseteq \subseteq^{e s s} R_{R}$.

Proof.(1) By Corollary 5.2 and [14, Lemma 2.4].

(2) Let $K$ be a right ideal of $R$ and $r(J) \cap K=0$. Then $\operatorname{Kr}(J)=0$ and we obtain $K \subseteq \operatorname{lr}(J)=J$, because $R$ is left Kasch. By (1), we have $r(J+l(K))=r(J) \cap K=$ 0 and this means that $J+l(K)=R$ ( since $R$ is left Kasch). Thus $K=0$ and hence $r(J) \subseteq{ }^{e s s} R_{R}$.

The following examples show that the three classes of rings: strongly SS-injective rings, soc-injective rings and small injective rings are different. 
Example 5.5. Let $R=\mathbb{Z}_{(p)}=\left\{\frac{m}{n}: p\right.$ does not divide $n$, the localization ring of $\mathbb{Z}$ at the prime $p$. Then $R$ is a commutative local ring and it has zero socle but not principally small injective (see [4, Example 4]). Since $S_{r}=0$, thus $R$ is strongly soc-injective ring and hence $R$ is strongly SS-injective ring.

Example 5.6. Let $R=\left\{\left(\begin{array}{ll}n & x \\ 0 & n\end{array}\right): n \in \mathbb{Z}, x \in \mathbb{Z}_{2}\right\}$. Thus $R$ is a commutative ring, $J=S_{r}=\left\{\left(\begin{array}{ll}0 & x \\ 0 & 0\end{array}\right): x \in\right.$ $\left.\mathbb{Z}_{2}\right\}$ and $R$ is small injective ( see [3, Example (i)]). Let $A=J$ and $B=\left\{\left(\begin{array}{cc}2 n & 0 \\ 0 & 2 n\end{array}\right): n \in \mathbb{Z}\right\}$, then $l(A)=$ $\left\{\left(\begin{array}{cc}2 n & y \\ 0 & 2 n\end{array}\right): n \in \mathbb{Z}, y \in \mathbb{Z}_{2}\right\}$ and $l(B)=\left\{\left(\begin{array}{ll}0 & y \\ 0 & 0\end{array}\right): \quad y \in \mathbb{Z}_{2}\right\} . \quad$ Thus $\quad l(A)+l(B)=$ $\left\{\left(\begin{array}{cc}2 n & y \\ 0 & 2 n\end{array}\right): n \in \mathbb{Z}, y \in \mathbb{Z}_{2}\right\}$. Since $A \cap B=0$, then $l(A \cap B)=R$ and this implies that $l(A)+l(B) \neq$ $l(A \cap B)$. Therefore $R$ is not strongly SS-injective and not strongly soc-injective by Proposition 5.1.

Example 5.7. Let $F=\mathbb{Z}_{2}$ be the field of two elements, $F_{i}=F$ for $i=1,2, \ldots, Q=\prod_{i=1}^{\infty} F_{i}, S=$ $\bigoplus_{i=1}^{\infty} F_{i}$. If $R$ is the subring of $Q$ generated by 1 and $S$, then $R$ is a von Neumann regular ring ( see [17, Example (1), p. 28]). Since $R$ is commutative, thus every simple $R$ - module is injective by $[9$, Corollary 3.73. Thus $R$ is $V$-ring and hence and hence $J(N)=0$ for every right $R$-module $N$. It follows from Corollary 3.9 that every $R$-module is a strongly SS-injective. In particular, $R$ is a strongly SS-injective ring. But $R$ is not soc-injective ( see [17, Example (1)]).

Example 5.8. Let $R=\mathbb{Z}_{2}\left[x_{1}, x_{2}, \ldots\right]$ where $\mathbb{Z}_{2}$ is the field of two elements, $x_{i}^{3}=0$ for all $i, x_{i} x_{j}=0$ for all $i \neq j$ and $x_{i}^{2}=x_{j}^{2} \neq 0$ for all $i$ and $j$. If $m=x_{i}^{2}$, then $R$ is a commutative, local, soc-injective ring with $J=$ $\operatorname{span}\left\{m, x_{1}, x_{2}, \ldots\right\}$, and $R$ has simple essential socle $J^{2}=\mathbb{Z}_{2} m$ ( see [1, Example 5.7]). It follows from [1, Example 5.7] that the $R$-homomorphism $\gamma: J \rightarrow R$ which is given by $\gamma(a)=a^{2}$ for all $a \in J$ with simple image can not extend to $R$, then $R$ is not simple $J$ injective and not small injective, so it follows from Corollary 5.2 that $R$ is not strongly SS-injective.

Recall that a ring $R$ is called right minsymmetric if $a R$ is simple, $a \in R$, implies that $R a$ is simple left ideal (see [2]). Every right mininjective ring is right minsymmetric by [2, Theorem 1.14].

Theorem 5.9. A ring $R$ is $Q F$ if and only if $R$ is a strongly right SS-injective and right noetherian ring with $S_{r} \subseteq{ }^{e s s} R_{R}$.

Proof. $(\Rightarrow)$ This is clear.

$(\Leftarrow)$ By Lemma $4.3(1), R$ is a right minsymmetric. It follows from [3, Lemma 2.2] that $R$ is right perfect. Thus, $R$ is strongly right soc-injective, by Theorem 3.13. Since $S_{r} \subseteq^{e s s} R_{R}$, so it follows from [1, Corollary 3.2 ] that $R$ is a self-injective and hence $R$ is $Q F$.
Corollary 5.10. For a ring $R$, the following statements are true:

(1) $\quad R$ is a semisimple if and only if $S_{r} \subseteq{ }^{e s s} R_{R}$ and every semisimple right $R$-module is strongly socinjective.

(2) $R$ is $Q F$ if and only if $R$ is a strongly right SSinjective, semiperfect with essential right socle and $R / S_{r}$ is noetherian as right $R$-module.

Proof. (1) Suppose that $S_{r} \subseteq^{\text {ess }} R_{R}$ and every semisimple right $R$-module is strongly soc-injective, then $R$ is a right noetherian right $V$-ring by [1, Proposition 3.12], so it follows from Corollary 3.9 that $R$ is a strongly right SS-injective. Thus $R$ is $Q F$ by Theorem 5.9. But $J=0$, so $R$ is a semisimple. The converse is clear.

(2) By [2, Theorem 2.9], $J=Z_{r}$. Since $R / Z_{2}^{r}$ is a homomorphic image of $R / Z_{r}$ and $R$ is a semilocal ring, thus $R$ is a right $t$-semisimple. By Corollary 3.19, $R$ is right noetherian, so it follows from Theorem 5.9 that $R$ is $Q F$. The converse is clear.

Theorem 5.11. A ring $R$ is $Q F$ if and only if $R$ is strongly right SS-injective, $l\left(J^{2}\right)$ is a countable generated left ideal, $S_{r} \subseteq{ }^{e s s} R_{R}$ and the chain $r\left(x_{1}\right) \subseteq$ $r\left(x_{2} x_{1}\right) \subseteq \cdots \subseteq r\left(x_{n} x_{n-1} \ldots x_{2} x_{1}\right) \subseteq \cdots$ terminates for every infinite sequence $x_{1}, x_{2}, \ldots$ in $R$.

Proof. $(\Rightarrow)$ Clear.

$(\Leftarrow)$ By [3, Lemma 2.2], $R$ is right perfect. Since $S_{r} \subseteq{ }^{e s s} R_{R}$, thus $R$ is right Kasch by [2, Theorem 3.7]. Since $R$ is a strongly right SS-injective, $R$ is a right simple $J$-injective, by Corollary 5.2. Now, by Proposition 5.4 (1) we have $\operatorname{rl}\left(S_{r} \cap J\right)=S_{r} \cap J$, so Corollary 4.10 (7) leads to $S_{r}=S_{\ell}$. By [5, Lemma 3.36 , p. 73], $S_{2}^{r}=l\left(J^{2}\right)$. The result now follows from [14, Theorem 2.18].

Remark 5.12. The condition $S_{r} \subseteq \subseteq^{e s s} R_{R}$ in Theorem 5.9 and Theorem 5.11 can be not be deleted, for example, $\mathbb{Z}$ is a strongly SS-injective noetherian ring but not $Q F$.

The following two results are extension of Proposition 5.8 in [1].

Corollary 5.13. A ring $R$ is $Q F$ ring if and only if it is left perfect, strongly left and right SS-injective ring.

Proof. By Corollary 5.2 and [14, Corollary 2.12].

Theorem 5.14. For a ring $R$, the following statements are equivalent:

(1) $R$ is a $Q F$ ring.

(2) $R$ is a strongly left and right SS-injective, right Kasch and $J$ is left $t$-nilpotent.

(3) $R$ is a strongly left and right SS-injective, left Kasch and $J$ is left $t$-nilpotent. 
Proof. $(1) \Rightarrow(2)$ and $(1) \Rightarrow(3)$ are clear.

(3) $\Rightarrow(1)$ Suppose that $x R$ is simple right ideal. Thus either $r l(x)=x R \subseteq{ }^{\oplus} R_{R}$ or $x \in J$. If $x \in J$, then $r l(x)=x R$ ( since $R$ is right minannihilator), so Theorem 3.4 implies that $r l(x) \subseteq{ }^{e s s} E \subseteq{ }^{\oplus} R_{R}$. Therefore, $r l(x)$ is an essential in a direct summand of $R_{R}$ for every simple right ideal $x R$. Let $K$ be a left maximal ideal of $R$. Since $R$ is a left Kasch, thus $r(K) \neq 0$ by [9, Corollary 8.28, p. 281]. Choose $0 \neq y \in r(K)$, so $K \subseteq l(y)$ and we conclude that $K=l(y)$. Since $R y \cong R / l(y)$, thus $R y$ is simple left ideal. But $R$ is a left mininjective ring, so $y R$ is a simple right ideal by [2, Theorem 1.14] and this implies that $r(K) \subseteq \subseteq^{e s s} e R$ for some $e^{2}=e \in R$ ( since $r(K)=$ $r l(y))$. Thus $R$ is semiperfect by [5, Lemma 4.1, p. 79] and hence $R$ is a left perfect ( since $J$ is left $t$-nilpotent), so it follows from Corollary 5.13 that $R$ is $Q F$.

$(2) \Rightarrow(1)$ is similar to proof of $(3) \Rightarrow(1)$.

Theorem 5.15. The $\operatorname{ring} R$ is $Q F$ if and only if $R$ is a strongly left and right SS-injective, left and right Kasch, and the chain $l\left(a_{1}\right) \subseteq l\left(a_{1} a_{2}\right) \subseteq \cdots \subseteq l\left(a_{1} a_{2} \ldots a_{n}\right) \subseteq$ ... terminates for every $a_{1}, a_{2}, \ldots \in Z_{\ell}$.

Proof. $(\Rightarrow)$ Clear.

$(\Leftarrow)$ By Proposition 5.4, $l(J)$ is essential in ${ }_{R} R$. Thus $J \subseteq Z_{\ell}$. Let $a_{1}, a_{2}, \ldots \in J$, we have $l\left(a_{1}\right) \subseteq l\left(a_{1} a_{2}\right) \subseteq$ $\cdots \subseteq l\left(a_{1} a_{2} \ldots a_{n}\right) \subseteq \cdots$. Thus there exists $k \in \mathbb{N}$ such that $l\left(a_{1} \ldots a_{k}\right)=l\left(a_{1} \ldots a_{k} a_{k+1}\right) \quad$ (by hypothesis). Suppose that $a_{1} \ldots a_{k} \neq 0$, so $R\left(a_{1} \ldots a_{k}\right) \cap l\left(a_{k+1}\right) \neq$ 0 ( since $l\left(a_{k+1}\right)$ is essential in ${ }_{R} R$ ). Thus $r a_{1} \ldots a_{k} \neq 0$ and $r a_{1} \ldots a_{k} a_{k+1}=0$ for some $r \in R$, a contradiction. So, $a_{1} \ldots a_{k}=0$ and hence $J$ is left $t$-nilpotent, so it follows from Theorem 5.14 that $R$ is $Q F$.

\section{References}

[1] I. Amin, M. Yousif and N. Zeyada, Soc-injective rings and modules, Comm. Algebra 33 (2005), 42294250 .

[2] W. K. Nicholson and M. F. Yousif, Mininjective rings, J. Algebra 187 (1997), 548-578.

[3] L.V. Thuyet and T. C. Quynh, On small injective rings and modules, J. Algebra and Its Applications 8 (2009), 379-387.
[4] Y. Xiang, Principally small injective rings, Kyungpook Math. J. 51 (2011), 177-185.

[5] W. K. Nicholson and M. F. Yousif, Quasi-Frobenius rings, Cambridge Tracts in Math., 158, Cambridge University Press, Cambridge, 2003.

[6] C. Lomp, On semilocal modules and rings, Comm. Algebra 27 (1999), 1921-1935.

[7] F. W. Anderson and K. R. Fuller, Rings and Categories of Modules, Springer-Verlag, Berlin-New York, 1974.

[8] F. Kasch, Modules and Rings, Academic Press, New York, 1982.

[9] T.Y. Lam, Lectures on Modules and Rings, GTM 189, Springer-Verlag, New York, 1999.

[10] D. S. Passman, A coruse in ring theory, AMS Chelsea Publishing, 2004.

[11] P. E. Bland, Rings and Their Modules, Walter de Gruyter \& Co., Berlin, 2011.

[12] L. Bican, T. Kepka and P. Neme, Rings, Modules and Preradicals, Marcel Dekker, Inc, New York, 1982.

[13] Y. Hirano, Rings whose simple modules have some properties, pp.63-76 in J. L. Chen, N. Q. Ding and H. Marubayashi, Advances in Ring Theory, Proceedings of the 4th China-Japan-Korea International Conference, World Scientific Publishing Co. Pte. Ltd., 2005.

[14] M. F. Yousif and Y. Q. Zhou, FP-injective, simpleinjective and quasi-Frobenius rings, Comm. Algebra 32 (2004), 2273-2285.

[15] B. Stenström, Rings of Quotients, SepringerVerlage, Berlin-Heidelberg-New York, 1975.

[16] Sh. Asgari, A. Haghany and Y. Tolooei, Tsemisimple modules and T-semisimple rings, Comm. Algebra 41 (2013), 1882-1902.

[17] N. Zeyada, S-injective modules and rings, Advances in Pure Math. 4 (2014), 25-33.

[18] W. K. Nicholson, Semiregular modules and rings, Canadian J. Math. 28 (1976), 1105-1120.

[19] N. Zeyada, S. Hussein and A. Amin, Rad-injective and almost-injective modules and rings, Algebra Colloquium 18 (2011), 411-418.

[20] S. H. Mohamed and B. J. Müller, Continuous and Discrete Modules, Cambridge University Press, Cambridge, 1990. 


\section{الموديولات والحلقات الاغمارية من النمط-SS}

$$
\begin{aligned}
& \text { عادل سالم تايه } \\
& \text { قسم الرياضيات } \\
& \text { كلية علوم الحاسوب وتكنلوجيا } \\
& \text { المعلومات القات } \\
& \text { جامعة القادسية }
\end{aligned}
$$

Email: adel.tayh@qu.edu.iq

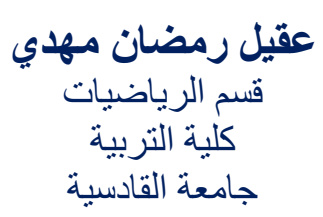

Email: akeel.mehdi@qu.edu.iq

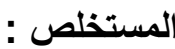

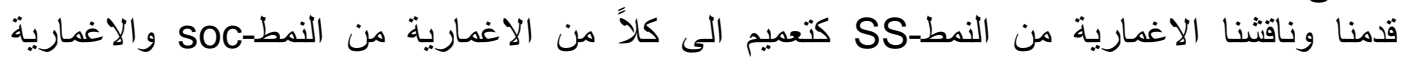

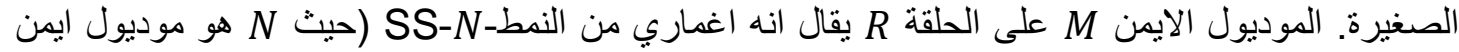

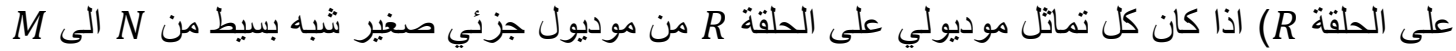

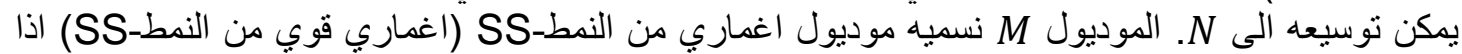

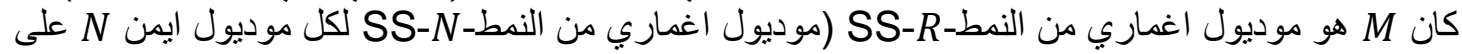

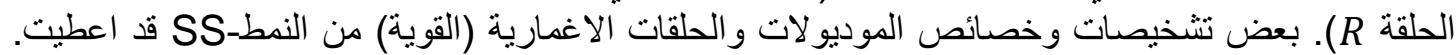

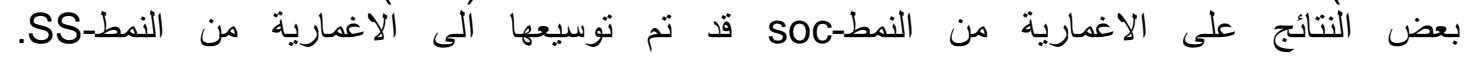

